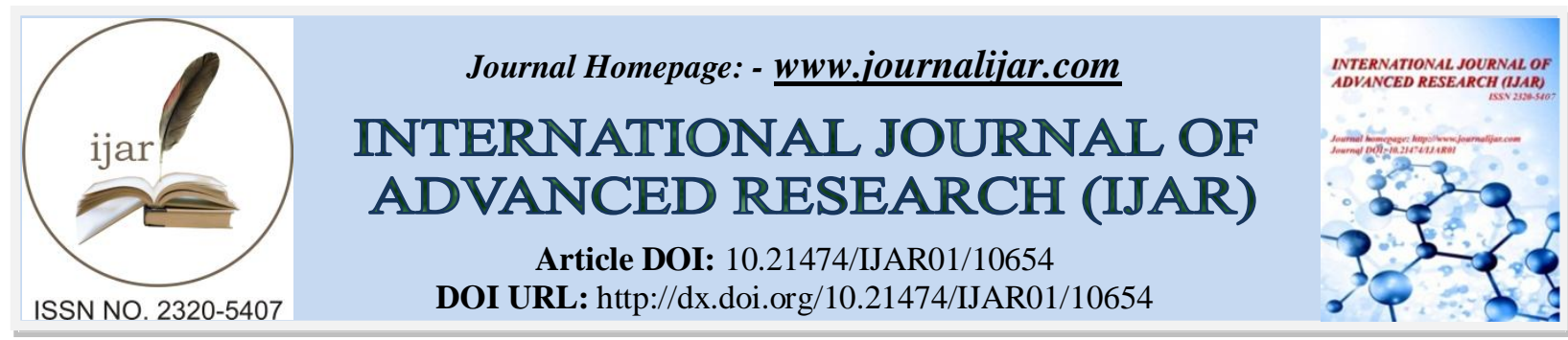

RESEARCH ARTICLE

\title{
TO STUDY THE INCIDENCE OF SYSTEMIC INFLAMMATORY RESPONSE SYNDROME (SIRS) OUTCOME IN PATIENTS UNDERGOING ELECTIVE OR EMERGENCY SURGERIES
}

\author{
Dr. Rupinder Singla ${ }^{1}$, Dr. Aastha Garg ${ }^{2}$ and Dr. Sanjeev Sharma ${ }^{3}$ \\ 1. Senior Resident, Department of Surgery, Fortis Hospital, Amritsar. \\ 2. Senior Resident, Department of Gynecology and Obstetrics, GMC, Amritsar. \\ 3. Associate Professor, Department of Surgery, GMC, Amritsar.
}

\section{Manuscript Info}

Manuscript History

Received: 10 January 2020

Final Accepted: 12 February 2020

Published: March 2020

\section{Key words:-}

Systemic Inflammatory Response Syndrome, Multi Organ Dysfunction Syndrome (MODS), Multi Organ Failure (MOF)

\section{Abstract}

Background: Systemic inflammatory response syndrome is a simple bed side tool to assess the severity of the patient's disease process which gives the approximate idea about the outcome of the surgical patients. Mortality rate increased progressively as the severity of systemic inflammatory response increased.This study investigated the Incidence of Systemic inflammatory response syndrome (SIRS) outcome in patients undergoing elective or emergency surgeries.

Objectives: To study the Incidence of Systemic inflammatory response syndrome (SIRS) outcome in patients undergoing elective or emergency surgeries

Methods: This was a prospective study conducted over a period of 2 years at GMC Amritsar. The total of 50 patients undergoing surgical procedures was studied. They were followed up till date of termination with daily SIRS monitoring, development of MODS and MOF.Statistical analysis was based on simple percentages among related variables.

Results: Overall incidence of SIRS was $74 \%$. Incidence of SIRS was more in patients undergoing emergency surgery than the patient who underwent elective surgery (43.47\%). The difference was statistically significant. $(p<0.05)$. Average SIRS score was higher in emergency surgery group than elective surgery group.

Conclusion: SIRS is key pathogenic factor in postoperative morbidity which gives the approximate idea about the outcome of the surgical patients.

Copy Right, IJAR, 2020,. All rights reserved.

\section{Introduction:-}

Despite continuous advances in anaesthesia, surgery and perioperative care, major surgery is associated with undesirable sequelae such as pain, cardiopulmonary, infective and thromboembolic complications, cerebral dysfunction, nausea and gastro-intestinal paralysis, fatigue and prolonged convalescence. Clearly, such morbidity may be related to the level of anaesthetic and surgical skill, but complications may occur regardless of skill and no single technique or drug regimen has been shown to eliminate postoperative morbidity and mortality. The key pathogenic factor in postoperative morbidity, excluding failures of surgical and anaesthetic techniques, is the surgical stress response with subsequent increased demands on organ function. This phenomenon is also called systemic inflammatory response syndrome ${ }^{1}$ 
Systemic inflammatory response syndrome is defined as two or more of the following conditions:

1. Temperature greater than $38^{\circ} \mathrm{C}$ or less than $36^{\circ} \mathrm{C}$;

2. Heart rate greater than 90 beats $/ \mathrm{min}$;

3. Respiratory rate greater than 20 breaths/ min or PaCO2 less than 32 torr;

4. White blood cell (WBC) greater than $12,000 / \mu$ l or less than $4000 / \mu 1$ or greater than $10 \%$ immature (band) forms. ${ }^{2}$

The SIRS includes two general phrases-An acute pro inflammatory state resulting from innate immune system recognition of ligands and An anti- inflammatory phase that may serve to modulate pro inflammatory phase.Under normal conditions, these coordinated responses direct a return to homeostasis. ${ }^{3}$

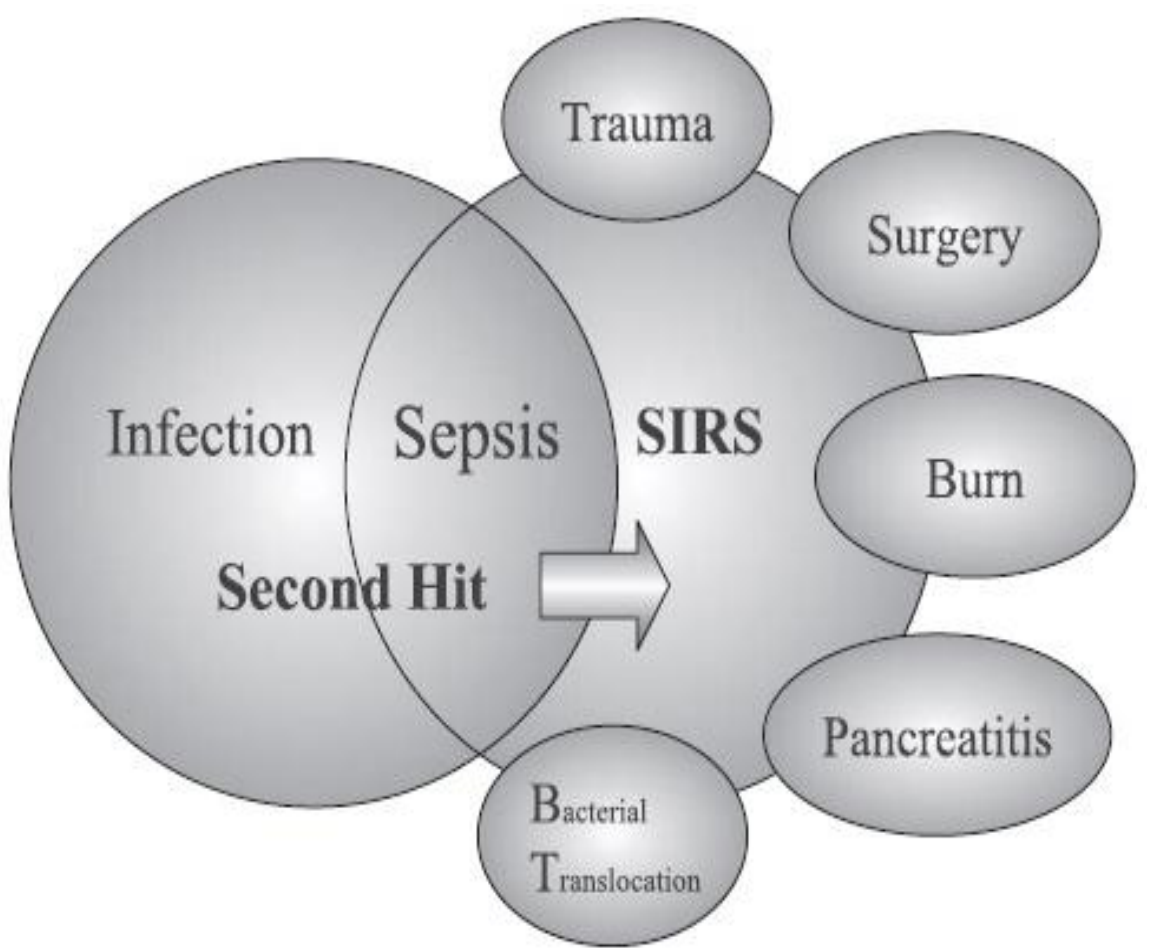

Fig 1:- The interrelationship between SIRS, sepsis, and infection.

SIRS leads to Multiorgan dysfunction could be a consequence of inconvenient clinical course of SIRS; the dysfunction is mostly related to the kidneys, liver, lungs, central nervous system and heart ${ }^{4}$.Vasodilatation, Increasedvascular permeability, Endothelial damage with expression of cell adhesion molecules and small thrombi in microcirculation - disseminated intravascular coagulation, Production of reactive oxygen species by neutrophils, Production of proteases by neutrophils, Production of NO by inductive NO synthase - refractoriness in vasodilatation. ${ }^{5}$ Resuscitation should be started as soon as the syndrome is recognised. Goal of initial resuscitation should be to maintain central venous pressure of $8-12 \mathrm{mmHg}$, mean arterial pressure of $\geq 65 \mathrm{mmHg}$, urine output $\geq 0.5 \mathrm{~mL} / \mathrm{Kg} /$ Hour and central venous oxygen saturation $\geq 70 \%$. Intravenous fluids, packed red blood cells and dobutamine infusion can be used for resuscitation. Intravenous antibiotics should be started within first hour of recognition of severe sepsis. Crystalloids and colloids should be used to correct hypovolemia.Intravenous corticosteroids (Hydrocortisone 200-300 mg/day, for 7 days in three or four divided doses) are recommended in patients with septic shock who, despite adequate fluid replacement, require vasopressor therapy to maintain blood pressure. Recombinant human activated protein C (rhAPC) is recommended in patients at high risk of death. ${ }^{6}$

\section{Aims and Objectives:-}

1. To study the incidence of Systemic inflammatory response syndrome outcome in 50 patients undergoing elective or emergency surgeries in Surgical Unit of Guru Nanak Dev Hospital, Amritsar.

2. Incidence of factors causing mortality and its relation with SIRS.

3. Average SIRS score in emergency and elective surgery 


\section{Material and methods:-}

This was a prospective study conducted over a period of 2 years. The total of 50 patients undergoing surgical procedures were studied. They were followed up till date of termination with daily SIRS monitoring, development of MODS and MOF. Risk factors of MOF were addressed.

Criteria for SIRS was kept as: Two or more of the following variables ${ }^{2}$

1. Pulse rate (PR): $>90$ beats $/ \mathrm{min}$

2. Respiratory Rate (RR): $>20$ breaths $/ \mathrm{min}$ or a $\mathrm{Pa} \mathrm{CO}_{2}$ level $<32 \mathrm{~mm}$ of $\mathrm{Hg}$

3. Temperature: $>38^{\circ} \mathrm{C}$ or $<36^{\circ} \mathrm{C}$

4. Total Leucocyte count (TLC): $>12,000 / \mu 1$ or $<4,000 / \mu 1$ or $>10 \%$ bands.

The SIRS scores were calculated daily and maximum score were recorded. The SIRS score assigns 1 point for each parameter (temperature, white blood cell count, heart rate, and respiratory rate), so that a maximum of 4 points can be accrued. A maximal SIRS score was calculated by summing the individual worst value in each of the 4 parameters. Thus, a patient might have a maximal SIRS score of 4 , even if the patient had scored 1 point in each different parameter at different points in the ICU admission. Pulse rate, Respiratory rate, temperature were recorded daily manually. TLC was done. Type of operation, type of anaesthesia, untoward incident during surgery and duration of surgery recorded. Co-morbid condition, Focus of sepsis was looked for and ventilator support was considered as a positive SIRS criteria for respiration.

\section{Results:-}

Incidence of sirs in elective and emergency surgery:

\begin{tabular}{|c|c|c|c|}
\hline Type of Surgery & Total No. of Patients & No. of Patients with SIRS & No. of Patients without SIRS \\
\hline Elective & 23 & 10 & 13 \\
\hline Emergency & 27 & 27 & 0 \\
\hline Total & 50 & 37 & 13 \\
\hline
\end{tabular}

Table 1 showing incidence of systemic inflammatory response syndrome in elective and emergency surgery. SIRS developed in 37 patients (74\%). All the patients in emergency surgery group had SIRS at some point of time during hospital stay. 10 patients $(43.47 \%)$ from elective surgery group developed SIRS. Incidence of SIRS was more in patients undergoing emergency surgery than the patient who underwent elective surgery. The difference was statistically significant. $(\mathrm{p}<0.05)$.

Average sirs score in emergency and elective surgery:

\begin{tabular}{|c|c|c|c|}
\hline Average SIRS Score & No. of Patients in Emergency Surgery & No. of Patients in Elective Surgery & Total \\
\hline $0-1$ & 10 & 20 & 30 \\
\hline $1-2$ & 13 & 2 & 15 \\
\hline $2-3$ & 1 & 0 & 1 \\
\hline $3-4$ & 3 & 1 & 4 \\
\hline Total & 27 & 23 & 50 \\
\hline
\end{tabular}

Table 2 showing average SIRS score in emergency and elective surgery. Most of the patients in emergency surgery group were having mean SIRS score in 1-2 range and most of patients undergoing elective surgery were having mean SIRS score in 0-1 range group. Average SIRS score was more in emergency surgery group than in elective surgery group.

Incidence of mortality:

\begin{tabular}{|c|c|}
\hline Diagnosis & Incidence of Mortality \\
\hline Overall Mortality & $10 \%$ \\
\hline SIRS & $13.51 \%$ \\
\hline Sepsis & $45.45 \%$ \\
\hline Severe Sepsis & $83.33 \%$ \\
\hline MODS & $100 \%$ \\
\hline
\end{tabular}

Table 3 showing incidence of mortality. Overall mortality in our study was 10\%. Rate of mortality in patients who developed systemic inflammatory response syndrome was $13.51 \%$. Mortality rate increased to $45.45 \%$ in patients who developed sepsis. In case of patients with severe sepsis mortality rate was as high as $83.33 \%$. The patients who 
developed multi organ dysfunction were having mortality rate of $100 \%$. Mortality increased progressively from SIRS, sepsis, severe sepsis, MODS respectively.

\section{Discussion:-}

In our study SIRS developed in 37 patients (74\%). Similar observations were made in study conducted by RangelFrausto et $\mathrm{al}^{7}$ who found that $85.7 \%$ patients in surgical intensive care units (SICU) had systemic inflammatory response syndrome.

Overall mortality in our study was $10 \%$. Rate of mortality in patients who developed systemic inflammatory response syndrome was $13.51 \%$. Mortality rate increased to $45.45 \%$ in patients who developed sepsis. In case of patients with severe sepsis mortality rate was as high as $83.33 \%$. The patients who developed multi organ dysfunction were having mortality rate of $100 \%$. Mortality increased progressively from SIRS, sepsis, severe sepsis, MODS respectively. Pittet et $\mathrm{al}^{8}$ had made similar observations with 83 patients (49\%) having sepsis; among them 28 developed severe sepsis. Rangel-Frausto et $\mathrm{al}^{7}$ had seen that among patients with SIRS, 649 (26\%) developed sepsis, 467 (18\%) developed severe sepsis, and $110(4 \%)$ developed septic shock and, Therewere also stepwise increases in mortality rates in the hierarchy from SIRS, sepsis, severe sepsis, and septic shock: $7 \%, 16 \%, 20 \%$, and $46 \%$, respectively.

\section{Summary and Conclusion:-}

$27(54 \%)$ patients were operated as emergency surgical procedure and $23(46 \%)$ patients were operated as elective surgical procedure.Overall incidence of SIRS was 74\%.Patients undergoing emergency surgery were having higher incidence of SIRS than the patients undergoing elective surgery. Any particular importance could not be attributed to any of the SIRS criteria individually.SIRS criteria is a simple, easy to do bed side/laboratory. SIRS is an important response to surgical trauma with high mortality and morbidity.Mortality, duration of hospital, Multiorgan dysfunction and ICU stayincreased significantly in the patients having SIRS. When SIRS is present always look for focus of infection like wound, urinary tract infections, lungs etc.SIRS therefore assumes importance, because when it is present, it alerts to look for septic foci or second hit.

\section{References:-}

1. Comstedt P, Storgaard M and Lassen AT. The Systemic Inflammatory Response Syndrome (SIRS) in acutely hospitalised medical patients: a cohort study. Scandinavian Journal of Trauma, Resuscitation and Emergency Medicine 2009; 17:67.

2. Singh S, Singh P, Singh G. Systemic inflammatory response syndrome outcome in surgical patients. Indian J Surg. 2009 Aug; 71(4):206-9.

3. Brun-Buisson C. The epidemiology of the systemic inflammatory response. Intensive Care Medicine. 2000; 26(1):S064-S074.

4. Baue AE. MOF, MODS, and SIRS: what is in a name or an acronym? Shock. 2006 Nov;26(5):438-49.

5. Jaffer U, Wade RG, Gourlay T. Cytokines in the systemic inflammatory response syndrome: a review. HSR Proceedings in Intensive Care and Cardiovascular Anesthesia 2010; 2(3): 161-175.

6. Dellinger RP, Carlet JM, Masur H, Gerlach H, CalandraT, Cohen J et al. Surviving sepsis campaign guidelines for management of severe sepsis and septic shock. Crit Care Med. 2004; 32(3):858-73.

7. Rangel-Frausto MS, Pittet D, Costigan M, Hwang T, Davis CS, Wenzel RP. The Natural History of the Systemic Inflammatory Response Syndrome (SIRS)- A Prospective Study.JAMA.1995; 273(2):117-23.

8. Pittet D, Rangel-Frausto S, Li N, Tarara D, et al. Systemic inflammatory response syndrome, sepsis, severe sepsis and septic shock: Incidence, morbidities and outcomes in surgical ICU patients. Intensive Care Med. 1995 Apr; 21(4):302-9. 